\title{
O papel dos gestos no desenvolvimento da linguagem oral de crianças com desenvolvimento típico e crianças com síndrome de Down
}

\author{
The role of gestures in oral language development of typically \\ developing children and children with Down syndrome
}

\author{
Fabíola Custódio Flabiano-Almeida ${ }^{1}$, Suelly Cecilia Olivan Limongi²
}

\begin{abstract}
RESUMO
O objetivo do presente estudo foi caracterizar o papel dos gestos nos estágios iniciais do desenvolvimento da linguagem oral de crianças com desenvolvimento típico e crianças com síndrome de Down (SD), a partir de amplo levantamento bibliográfico em bases de dados, abrangendo as duas últimas décadas de estudos na área. A literatura pesquisada sugere que os gestos desempenham papel importante no desenvolvimento da linguagem oral, fornecendo à criança recursos cognitivos extras para a aprendizagem de novas palavras e enunciados. É apontada também a função social dos gestos nesse processo, na medida em que sinalizam ao interlocutor que a criança já está pronta para receber determinado input linguístico, eliciando produções verbais do adulto que fornecem à criança o modelo de como expressar suas ideias inteiramente por meio da fala. Com relação às crianças com SD, os gestos também parecem ser preditivos do desenvolvimento lexical; porém, apenas em relação ao vocabulário receptivo, enquanto as dificuldades específicas relatadas em relação à transição das combinações de gesto e palavra para as combinações de duas ou mais palavras têm sido especuladas como o primeiro indício de futuros déficits no desenvolvimento da sintaxe, frequentemente observados nessa população. Além disso, os estudos na área têm sugerido que características da interação comunicativa entre mãe e criança com SD, tais como a produção de gestos de forma breve e confusa pelas crianças e menor responsividade de suas mães, também podem contribuir para as dificuldades de expressão verbal observadas nessas crianças.
\end{abstract}

Descritores: Gestos; Desenvolvimento; Linguagem oral; Crianças; Síndrome de Down

\section{INTRODUÇÃO}

Os gestos são definidos como ações produzidas para fins de comunicação, geralmente realizados usando-se os dedos, mãos e braços, mas podendo também incluir movimentos faciais e corporais ${ }^{(1)}$. Tradicionalmente, os gestos são divididos em duas categorias: dêiticos e representativos. Os dêiticos seriam aqueles utilizados para estabelecer um referencial, indicando

Trabalho realizado no Departamento de Fisioterapia, Fonoaudiologia e Terapia Ocupacional da Faculdade de Medicina da Universidade de São Paulo - USP - São Paulo (SP), Brasil, com bolsa concedida pela Fundação de Amparo à Pesquisa do Estado de São Paulo - FAPESP.

(1) Doutora, Fonoaudióloga pesquisadora do Laboratório de Investigação Fonoaudiológica em Síndromes e Alterações Sensóriomotoras do Departamento de Fisioterapia, Fonoaudiologia e Terapia Ocupacional da Faculdade de Medicina da Universidade de São Paulo - USP - São Paulo (SP), Brasil. (2) Livre-docente, Professora do Curso de Fonoaudiologia do Departamento de Fisioterapia, Fonoaudiologia e Terapia Ocupacional da Faculdade de Medicina da Universidade de São Paulo - USP - São Paulo (SP), Brasil.

Endereço para correspondência: Fabíola Custódio Flabiano-Almeida. R. Cipotânea, 51, Cidade Universitária, São Paulo (SP), Brasil, CEP: 05360-160.

E-mail: fabiolaflabiano@yahoo.com.br

Recebido em: 23/5/2010; Aceito em: 27/7/2010 um objeto ou evento ${ }^{(2)}$ e, portanto, sua interpretação depende do contexto em que é realizado. Já os gestos representativos apresentam conteúdo semântico específico. Tais gestos podem se referir a objetos, significando alguma de suas características (gestos simbólicos), como por exemplo, o gesto de abrir e fechar a mão em frente à boca (comer), ou ser definidos culturalmente (gestos convencionais), como por exemplo, os gestos de dar tchau e de mandar beijo, em que não há um objeto ou ação específica a ser representada.

Os gestos dêiticos são a primeira manifestação da comunicação intencional e sua sequência de desenvolvimento revela um gradual distanciamento do objeto concreto, seguindo na mesma direção do desenvolvimento simbólico ${ }^{(3)}$. Assim, no início do desenvolvimento, a criança apenas mostra o objeto em sua mão, estendendo o braço e olhando para o adulto. Mas logo passa a entregá-lo, o que constitui o "dar". A criança, então, começa a utilizar o estender de braço para pedir objetos próximos a ela e, mais tarde, começa a utilizar o apontar para objetos ou eventos que estão distantes ou fora do seu alcance, seja para pedi-los ou para mostrá-los. Nessa mesma fase, quando a criança torna-se capaz da imitação diferida e 
da representação; são observados os primeiros gestos representativos e esquemas simbólicos, geralmente combinados às primeiras palavras.

De acordo com a literatura ${ }^{(4-5)}$, as primeiras combinações de gesto e palavra observadas na criança são combinações de reforço, em que o gesto reforça a informação contida na palavra; como por exemplo, quando a criança diz "tchau", ao mesmo tempo em que realiza o gesto de dar tchau. A seguir, aparecem as combinações complementares, em que o gesto serve para identificar o referente a ser nomeado por meio da palavra, como por exemplo, a criança aponta para o carro e diz "carro". Meses depois, a criança começa a produzir combinações de gesto e palavra em que o gesto traz uma informação suplementar à informação contida na palavra, como por exemplo, quando a criança aponta para a banana e diz "comer".

As combinações suplementares permitem à criança expressar dois elementos semânticos de uma sentença (um em forma de gesto e o outro em forma de palavra), e, por isso, são consideradas por vários autores como precursores das primeiras sentenças formadas por duas ou mais palavras ${ }^{(4-7)}$.

Nas últimas décadas, muitos estudos têm investigado o valor preditivo dos gestos, tanto em relação aos aspectos lexicais quanto em relação aos aspectos sintáticos da linguagem. As evidências têm mostrado que os gestos funcionam não apenas como elemento de transição entre as ações motoras e a linguagem oral, mas também como facilitador do processo de produção da fala, fornecendo à criança, nos estágios iniciais do desenvolvimento da linguagem, recursos cognitivos extras, que permitem representar e comunicar ideias mais complexas, enquanto ainda não conseguem fazê-lo exclusivamente por meio da fala ${ }^{(5)}$.

Nesse sentido, alguns pesquisadores também têm buscado compreender como se dá a relação entre o desenvolvimento dos gestos e a emergência da linguagem oral em crianças com síndrome de Down (SD). Embora ainda escassas, as pesquisas existentes nessa área têm mostrado que o desenvolvimento dos gestos e da linguagem oral parece acontecer de forma similar ao observado no desenvolvimento típico (DT) durante os primeiros estágios do desenvolvimento comunicativo na criança com SD. Porém, a natureza da relação entre gestos e linguagem começa a se modificar conforme a criança vai progredindo em seu desenvolvimento e as habilidades cognitivas vão se tornando melhores que as habilidades de linguagem expressiva ${ }^{(8)}$.

O objetivo do presente estudo foi caracterizar o papel dos gestos nos estágios iniciais do desenvolvimento da linguagem oral de crianças com DT e crianças com SD, a partir de um amplo levantamento bibliográfico em bases de dados (SciELO, LILACS, PubMed, Dedalus), que abrangeu as duas últimas décadas de estudos na área.

\section{REVISÃO DA LITERATURA}

\section{Valor preditivo dos gestos para o desenvolvimento lexical}

A relação entre o uso de gestos e o desenvolvimento lexical tem sido amplamente estudada. Pesquisas recentes têm demonstrado que os gestos não apenas precedem, mas também são preditivos do vocabulário, tanto expressivo quanto recep- tivo, em crianças com $\mathrm{DT}^{(9-11)}$.

Estudo anterior ${ }^{(9)}$ demonstrou que os itens encontrados inicialmente no repertório gestual de crianças com DT foram observados em seu vocabulário expressivo três meses após terem sido observados em forma de gestos.

Outros autores ${ }^{(12)}$ observaram que as crianças que utilizavam o gesto de apontar aos 11 meses apresentaram vocabulário mais amplo aos dois anos do que as crianças que ainda não apontavam aos 11 meses.

Pesquisadores $^{(13)}$ encontraram que a diversidade de significados representados por meio de gestos por crianças com DT aos 14 meses estava significantemente relacionada ao tamanho de seu vocabulário receptivo aos 42 meses. Além disso, os achados de um estudo posterior ${ }^{(10)}$ revelaram que o efeito do nível sócio-econômico no desenvolvimento do vocabulário de crianças aos 54 meses de idade foi parcialmente relacionado à sua produção gestual aos 14 meses.

A mesma relação preditiva entre os gestos e o desenvolvimento do vocabulário tem sido encontrada para crianças consideradas de risco para alterações no desenvolvimento de linguagem.

Alguns autores ${ }^{(13)}$ investigaram o uso de gestos em crianças com atraso de linguagem com idades entre 18 e 28 meses. Os resultados revelaram que as crianças que apresentaram normalização do vocabulário expressivo um ano depois foram as mesmas que haviam produzido maior diversidade de gestos comunicativos. Outros pesquisadores ${ }^{(14)}$ também encontraram déficits no uso de gestos em crianças com distúrbio específico de linguagem.

Estudo recente ${ }^{(15)}$ a respeito da relação entre os gestos e o vocabulário em crianças com lesões cerebrais pré ou peri-natais demonstrou que as crianças que produziram menor diversidade de gestos aos 18 meses também apresentaram escores mais baixos nas provas de vocabulário receptivo e expressivo aos 30 meses.

Portanto, o valor preditivo dos gestos para o desenvolvimento lexical parece ser válido não apenas para as crianças em DT, mas também para crianças com alterações do desenvolvimento de linguagem.

Alguns autores sugerem que, por meio dos gestos, a criança tem a oportunidade de se referir a objetos cujos nomes ainda não consegue expressar verbalmente. Assim, o uso constante de gestos com propósitos comunicativos forneceria à criança um meio de aprender e expressar diferentes significados, favorecendo a ampliação de seu vocabulário ${ }^{(9)}$.

Portanto, os gestos podem servir não apenas como um importante indicador do desenvolvimento lexical, mas podem também desempenhar papel fundamental na aprendizagem de novos vocábulos ${ }^{(16)}$.

\section{Uso de gestos e o desenvolvimento lexical na criança com SD}

Muitos estudos têm sugerido que as crianças com SD apresentam preferência pelo meio comunicativo gestual ao meio verbal ${ }^{(17-19)}$ em função das dificuldades na linguagem oral, especialmente em relação aos aspectos fonológicos ${ }^{(20)} \mathrm{e}$ morfossintáticos ${ }^{(21)}$. 
Diversos autores têm observado que durante o período de expansão do vocabulário, as crianças com $\mathrm{SD}$, ao invés de irem progressivamente substituindo os gestos pelas palavras correspondentes em suas produções, como acontece com a criança com DT, utilizam os gestos simultaneamente às palavras ou ainda utilizam as palavras como suporte aos gestos, e não o contrário ${ }^{(8,17,19,22)}$.

A grande variabilidade individual em relação ao desenvolvimento da linguagem em crianças com SD também tem sido relatada na literatura.

Pesquisadores ${ }^{(19)}$ estudaram a emergência da comunicação expressiva em crianças com SD e observaram grande variabilidade entre os sujeitos. Algumas crianças inicialmente apresentaram uso dos gestos simultaneamente às palavras e, com o desenvolvimento lexical, houve diminuição do uso de gestos e aumento da produção de palavras, enquanto outras apresentaram aumento do repertório gestual, em detrimento da linguagem oral.

A existência de uma "vantagem", pelas crianças com $\mathrm{SD}$, com respeito ao uso de gestos, é controversa segundo a literatura. Estudo realizado a partir de questionários aplicados aos pais ${ }^{(23)}$ demonstrou repertório gestual mais extenso em crianças com SD, quando comparadas a crianças com DT pareadas em relação ao vocabulário receptivo e expressivo. Resultados semelhantes foram posteriormente encontrados por outro estudo ${ }^{(24)}$.

No entanto, pesquisas mais recentes, que utilizam métodos mais precisos de pareamento dos sujeitos, têm indicado resultados contrários.

Pesquisadores ${ }^{(8)}$ examinaram o uso de gestos e palavras em crianças com SD e crianças com DT pareadas pela idade linguística, em termos da diversidade e frequência das produções. Os autores encontraram produções com número e frequência semelhantes nos dois grupos, tanto em relação aos gestos quanto em relação às palavras produzidas. Além disso, as crianças com SD apresentaram repertório mais reduzido de gestos representativos do que as crianças com DT em mesma fase de desenvolvimento linguístico.

Outros autores ${ }^{(25)}$ estudaram a produção de gestos e palavras durante uma prova de nomeação em 15 crianças com SD e dois grupos de crianças com DT, sendo um grupo pareado pela idade cronológica e o outro pela idade mental. Observou-se uso de gestos com frequência semelhante entre as crianças com SD e seus pares de mesma idade mental. Porém, as crianças com DT utilizaram os gestos acompanhados das designações verbais corretas, enquanto as crianças com SD tenderam a utilizar os gestos de forma isolada na tarefa de nomeação.

Em outro estudo ${ }^{(26)}$ foi encontrada correlação positiva e significativa entre a produção de gestos comunicativos aos 36 meses e o desenvolvimento lexical aos 42 meses em crianças com SD. Porém, tal relação foi obtida apenas para o vocabulário receptivo. Portanto, parece que, apesar dos gestos também serem preditivos do desenvolvimento lexical na criança com $\mathrm{SD}$, eles parecem estar mais ligados à compreensão das palavras do que à sua expressão oral.

Alguns autores têm argumentado que os gestos requerem menor demanda de memória do que as palavras, cujas formas convencionalizadas precisam ser memorizadas e evocadas no momento da produção. Os gestos dêiticos, cuja forma não varia conforme o referente, seriam ainda mais fáceis de memorizar e reproduzir, exigindo menor esforço cognitivo ${ }^{(27)}$. Além disso, o uso de gestos manuais convencionais requer menor controle motor fino do que os movimentos articulatórios necessários para a produção dos fonemas ${ }^{(28)}$.

Portanto, na criança com SD, assim como na criança com DT, os gestos parecem constituir um meio para o aprendizado de novos significados, dando à criança a possibilidade de praticar a comunicação desses novos significados, de forma a prepará-la para expressá-los mais tarde por meio da linguagem oral.

Todavia, a expressão verbal desses significados parece depender de outros fatores envolvidos no processo de desenvolvimento da linguagem oral ${ }^{(26)}$, tais como a memória fonológica e a memória de curto prazo, aspectos práxicos e de motricidade fina, para os quais as crianças com SD geralmente apresentam dificuldades ${ }^{(29)}$ e que, portanto, contribuem de forma significativa para que essas crianças utilizem o meio gestual como principal meio de comunicação durante longos períodos de tempo.

\section{Valor preditivo dos gestos para o desenvolvimento inicial da sintaxe.}

Vários estudos têm demonstrado evidências de que a idade em que a criança começa a produzir combinações suplementares de gesto e palavra é preditiva da idade em que a criança dará início à produção de combinações de duas palavras ${ }^{(4,7,9,28,30)}$. Assim, as combinações suplementares de gesto e palavra parecem anunciar que a criança logo começará a produzir combinações de duas palavras.

Pesquisadores ${ }^{(4)}$ encontraram que as primeiras combinações de duas palavras foram observadas por volta dos 17 meses, logo após o início das combinações suplementares de gesto e palavra (por volta dos 16 meses) em um grupo de dez crianças com DT.

Outros autores ${ }^{(30)}$ também encontraram que crianças com DT, mesmo de diferentes culturas (americana e italiana), começaram a produzir combinações suplementares de gesto e palavra aos 16 meses de idade, sendo que as combinações de duas palavras emergiram em média dois meses mais tarde, corroborando os achados de pesquisa anterior ${ }^{(9)}$, cujos autores verificaram ainda que as combinações de duas palavras eram correspondentes em significado às combinações de gesto e palavra produzidas anteriormente pelas crianças. Assim, por exemplo, a combinação de gesto e palavra "apontar para o carro + papai" foi posteriormente observada na forma da combinação de duas palavras "carro papai".

Estudos recentes têm sugerido que o papel dos gestos no processo de transição das combinações de gesto e palavra para as combinações de duas palavras consiste no fato de que as combinações de gesto e palavra permitem que a criança comunique dois significados diferentes de forma relacionada, dando à criança a possibilidade de praticar construções linguísticas mais complexas, enquanto ainda não consegue realizar tais construções exclusivamente por meio da fala ${ }^{(6,9,28,30)}$. 


\section{Gestos e o desenvolvimento inicial da sintaxe em crianças com SD}

Estudos anteriores têm demonstrado atrasos significativos em relação ao início da produção de combinações de duas ou mais palavras pelas crianças com SD, em comparação com as crianças com DT de mesma idade mental ${ }^{(8,31,32)}$. Além disso, são observadas sentenças mais curtas e de menor complexidade sintática, nas quais artigos, pronomes e preposições, entre outros, são omitidos ${ }^{(21)}$.

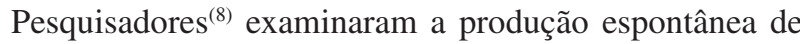
gestos e palavras por cinco crianças com SD, comparadas a cinco crianças com DT de mesma idade mental. Com relação aos tipos de combinações de gesto e palavra apresentados, as crianças com $\mathrm{SD}$ apresentaram duas vezes mais combinações de reforço e metade do número de combinações complementares. Além disso, a produção de combinações suplementares, cognitivamente mais sofisticadas, foi escassa no grupo de crianças com SD, enquanto as crianças com DT apresentaram número significativo dessas combinações, além de algumas combinações de duas palavras, não observadas nas crianças com SD.

Estudo realizado em instituição nacional ${ }^{(33)}$ com o objetivo de evidenciar a relação entre os gestos e o desenvolvimento da linguagem oral em um par de gêmeos monozigóticos com SD revelou que, mesmo após a emergência da linguagem oral, os sujeitos continuaram comunicando-se preferencialmente por gestos, utilizando algumas combinações de gesto e palavra. Porém, não foram observadas combinações de duas ou mais palavras.

Portanto, as crianças com SD parecem apresentar alguma dificuldade específica no processo de transição das combinações de gesto e palavra para as combinações de duas palavras, especialmente em relação às combinações suplementares, as quais são consideradas preditores confiáveis das primeiras combinações de duas ou mais palavras.

Uma possível explicação é a de que a emergência das combinações de duas palavras não apenas requer a habilidade cognitiva de combinar dois elementos semânticos, mas depende também de habilidades linguísticas adicionais que podem estar atrasadas ou comprometidas nas crianças com $\mathrm{SD}^{(29)}$. Se essas habilidades linguísticas forem apenas atrasadas, o intervalo entre o início das combinações suplementares de gesto e palavra e o início da produção de combinações de duas palavras seria apenas mais longo, ou seja, as crianças com SD precisariam de um período de tempo maior para desenvolver tais habilidades linguísticas específicas. Porém, se estas habilidades linguísticas estiverem comprometidas, a presença de combinações suplementares de gesto e palavra não teria o mesmo valor preditivo do início das combinações de duas palavras para as crianças com SD, como observado em crianças com DT.

Além disso, as dificuldades específicas em relação à transição das combinações de gesto e palavra e as combinações de duas palavras encontradas nas crianças com $\mathrm{SD}^{(8,17)}$ podem ser o primeiro indício de futuros déficits no desenvolvimento da sintaxe, frequentemente apresentado por essas crianças.

Todavia, outras pesquisas são necessárias para se verificar a relação entre as combinações de gesto e palavra e a produção de sentenças nas crianças com SD, em estágios posteriores do desenvolvimento cognitivo e da linguagem expressiva, a fim de se confirmar o valor preditivo das combinações de gesto e palavra nessa população, assim como observado nas crianças com DT.

\section{Função social dos gestos no desenvolvimento da linguagem}

Estudos recentes têm sugerido que, além do importante papel dos gestos no desenvolvimento da linguagem oral, permitindo à criança comunicar novos significados de forma isolada ou combinada e, portanto, fornecendo-lhe o potencial necessário para a aprendizagem de novas palavras e suas combinações ${ }^{(6,28,30)}$, os gestos também desempenhariam função social nesse processo, na medida em que sinalizam ao interlocutor que a criança já está pronta para receber determinado tipo de input linguístico ${ }^{(9)}$. O interlocutor, por sua vez, ao traduzir as produções gestuais da criança, fornecer-lhe-ia o modelo de como expressar suas ideias inteiramente por meio da fala ${ }^{(28,34)}$ (por exemplo, em resposta ao gesto da criança de apontar para uma boneca, a mãe provavelmente dirá "a boneca", ou se a criança apontar para a bolsa da mãe e disser "mamãe", a mãe provavelmente dirá "é, a bolsa da mamãe").

Pesquisadores $^{(34)}$ realizaram um estudo com o objetivo de investigar se as traduções que as mães faziam dos gestos realizados por suas crianças teriam alguma influência no seu desenvolvimento de linguagem. As díades foram observadas mensalmente dos dez aos 24 meses, em sessões de brincadeira livre com duração de 30 minutos cada. Os resultados revelaram que as mães traduziram em palavras ou sentenças os gestos e combinações de gesto e palavra produzidos por suas crianças. Além disso, as traduções realizadas pelas mães apresentaramse significativamente correlacionadas às produções verbais produzidas por suas crianças alguns meses depois.

Portanto, pode-se dizer que os gestos também desempenham papel indireto na aprendizagem de novas palavras ou sentenças, visto que eliciam produções verbais dos adultos, referentes ao objeto foco da atenção da criança ${ }^{(9)}$. Além disso, os gestos podem consistir em um meio de a criança direcionar o input linguístico que recebe, facilitando assim, seu próprio aprendizado $^{(34)}$.

Em relação à criança com SD, a literatura aponta que essas crianças geralmente apresentam dificuldades para prestar atenção a estímulos externos, são menos interativas e menos responsivas e, consequentemente, menos engajadas em atividades de jogo e brincadeira com seus pais ${ }^{(35)}$.

Alguns autores ${ }^{(17)}$ relataram que as crianças com SD produziram alguns gestos de forma súbita e breve, os quais foram difíceis de ser interpretados e respondidos pelas mães. Outros autores também relataram a produção de gestos de forma breve e confusa por crianças com SD como um fator que interfere negativamente nas interações com o adulto, resultando em desenvolvimento mais lento do vocabulário ${ }^{(36)}$.

Outros pesquisadores ${ }^{(37)}$ observaram que as mães de crianças com SD apresentaram comportamentos mais superficiais e menos sensíveis às tentativas de comunicação de seus filhos, 
quando comparadas às mães de crianças com DT aos 20 meses de idade.

Portanto, tais características das interações comunicativas entre mãe e criança com SD podem também contribuir para as dificuldades em relação à expressão verbal, amplamente relatadas nessa população, visto que interferem no recebimento do input adequado e favorável ao desenvolvimento da linguagem oral.

\section{DISCUSSÃO}

O presente estudo teve como objetivo caracterizar o papel dos gestos nos estágios iniciais do desenvolvimento da linguagem oral de crianças com DT e crianças com SD, a partir de um amplo levantamento bibliográfico, que abrangeu as duas últimas décadas de estudos na área.

A revisão de literatura revelou não apenas os principais achados a respeito da relação entre os gestos e o desenvolvimento da linguagem oral, mas também as questões que ainda necessitam de maiores esclarecimentos na área, especialmente ao se considerar as crianças com SD.

Os estudos realizados até o momento reforçam a ideia de que os gestos teriam valor preditivo para o desenvolvimento da linguagem, tanto em relação ao vocabulário receptivo e expressivo $^{(7,9-11)}$, quanto em relação ao início das combinações de palavras em sentenças ${ }^{(4,7,9,28-30)}$.

Assim, o estudo dos gestos nos estágios iniciais do desenvolvimento comunicativo pode consistir em um meio importante não apenas para a identificação de crianças com alterações de linguagem ${ }^{(38)}$, mas também para a distinção entre os casos de atraso e de distúrbio ${ }^{(13)}$.

Quanto às crianças com SD, estudos mais recentes também indicam o valor de predição dos gestos para o desenvolvimento lexical, embora relacionado apenas à compreensão ${ }^{(26)}$, e com o importante papel de favorecer o aprendizado de novos significados. Já em relação ao valor preditivo do uso de combinações suplementares de gestos e palavras para o uso de combinações de duas palavras, a literatura relata que as crianças com SD, além do atraso para o início da realização de tais combinações, também as apresentam com menor frequência e diversidade quando comparadas às crianças com $\mathrm{DT}^{(8,31-32)}$. Estudiosos na área justificam esses dados em função de as combinações suplementares de gesto e palavra serem cognitivamente mais sofisticadas e levantam a hipótese de que tais dificuldades seriam um primeiro indício de futuros déficits na produção sintática, geralmente apresentados pelas crianças com SD ${ }^{(8,17)}$. No entanto, ainda são necessárias outras pesquisas que estudem o valor preditivo das combinações de gesto e palavra para as combinações de duas ou mais palavras em estágios posteriores do desenvolvimento cognitivo e sintático de crianças com SD.

Assim, as crianças com SD frequentemente utilizam os gestos por um período de tempo mais longo, com o objetivo de serem melhor compreendidas pelo interlocutor ${ }^{(8,17,19)}$. A busca por justificativas para tais fatos indica a presença de outros aspectos que trariam dificuldades à expressão oral, como dificuldades de memória de curto prazo, memória fonológica, aspectos práxicos e de motricidade fina ${ }^{(29)}$ e que devem ser con- siderados ao se estabelecer a relação entre gestos e linguagem oral nessas crianças.

A literatura pesquisada também faz referência ao papel social dos gestos apresentados pelas crianças com DT em fase inicial de desenvolvimento de linguagem. Os estudos remetem para a importante participação do interlocutor nesse processo como tradutor dos gestos da criança e, nesse sentido, como favorecedor de seu desenvolvimento de linguagem ${ }^{(34)}$.

Com relação à criança com SD, apesar da forte presença dos gestos em sua comunicação, os estudos apontam resultados com mais reservas nessa área, devido a fatores que interferem no recebimento do input linguístico. Nesse sentido, são consideradas características presentes na criança com $\mathrm{SD}$, como a maneira breve e súbita de produzir os gestos; e outras presentes na mãe, como a pouca sensibilidade e responsividade às tentativas de comunicação do filho ${ }^{(35-37)}$.

Embora não tenha sido objeto tratado diretamente nos textos estudados, os achados apontados e comentados apresentam relevância à intervenção terapêutica em linguagem oral nas crianças com SD. Em relação ao processo de avaliação, a observação do uso dos gestos toma papel importante no auxílio da identificação de possíveis alterações na comunicação da criança. Alguns autores ${ }^{(39)}$ ressaltam a função comunicativa que os gestos devem desempenhar, atendendo aos aspectos de regulação do comportamento, de interação social e de atenção compartilhada. Outro aspecto ressaltado diz respeito às variações culturais e ao uso específico de gestos representativos próprios do ambiente familiar.

Tal observação é compartilhada por outros autores ${ }^{(30)}$, ao se referirem à intervenção terapêutica, e que também chamam a atenção para o aspecto da quantidade de gestos utilizados decorrentes da cultura e do meio familiar. Nesse sentido, a participação dos pais ou cuidadores no processo terapêutico, seja feito de forma direta ou por orientação, também é ressaltada em diferentes estudos ${ }^{(6,11,26,40)}$. Além da ênfase no uso de gestos combinados com vocalizações, palavras ou sentenças, outras pesquisas ressaltam a importância de sua utilização como fator de facilitação ao desenvolvimento da atenção compartilhada, fundamental para o desenvolvimento da linguagem e da comunicaçãa ${ }^{(34,39,40)}$.

A importância da interação pais/cuidadores-criança é comentada em estudos que apontam, além dos aspectos mencionados, o fato de que as combinações de gestos e expressão oral utilizados na interação entre adulto e criança favorecem a imitação por parte das crianças ${ }^{(6,10,11)}$, o que viria a ser fonte de compreensão verbal e de desenvolvimento lexical e sintático.

\section{COMENTÁRIOS FINAIS}

A revisão de literatura apresentada neste estudo mostrou grande variedade de achados relacionados ao papel dos gestos durante o processo de desenvolvimento da linguagem oral.

De maneira geral, os resultados sugerem que, por meio dos gestos, a criança tem a oportunidade de se referir a objetos cujos nomes ainda não consegue expressar verbalmente e de praticar a construção de estruturas sintáticas mais complexas, enquanto ainda não consegue expressá-las inteiramente por meio da fala. Assim, o uso de gestos isolados ou combinados a 
palavras forneceria à criança um meio de aprender e expressar diferentes significados e ideias, funcionando como ponte entre o vocabulário receptivo e o expressivo e entre as combinações de gesto e palavra e as combinações de palavras em sentenças. Além disso, os gestos também parecem desempenhar papel indireto no desenvolvimento da linguagem oral, na medida em que eliciam produções verbais dos adultos, referentes ao objeto foco da atenção da criança, fornecendo-lhe o modelo de como expressar suas ideias inteiramente por meio da fala.

Com relação à criança com $\mathrm{SD}$, apesar dos gestos também serem preditivos de seu desenvolvimento lexical, eles parecem estar mais ligados à compreensão das palavras do que à sua expressão oral. $\mathrm{O}$ valor preditivo das combinações de gesto e palavra para o desenvolvimento sintático nas crianças com
SD ainda não foi confirmado na literatura. Porém, dificuldades específicas em relação à transição das combinações de gesto e palavra para as combinações de duas palavras encontradas nessas crianças têm sido especuladas como o primeiro indício de futuros déficits no desenvolvimento da sintaxe, frequentemente observados nessa população.

Além disso, as dificuldades de manter a atenção em atividades compartilhadas e a produção de gestos de forma breve e confusa relatadas em crianças com SD, associadas a comportamentos menos sensíveis e responsivos de seus pais, também podem contribuir para as dificuldades de expressão verbal dessas crianças, visto que interferem no recebimento do input adequado e favorável ao desenvolvimento da linguagem oral.

\begin{abstract}
The aim of the present study was to characterize the role of gestures at the initial stages of oral language development in typically developing children and children with Down syndrome (DS), based on a large bibliographic review in scientific databases, covering the past two decades of studies on this subject. The researched literature suggests that the gestures play an important role in oral language development, providing the child with extra cognitive resources for the learning of new words and utterances. It has also been pointed out the social function of gestures in this process, since they sign to the interlocutor that the child is already ready to receive a particular linguistic input, eliciting verbal productions from the adult, who provide the child with the model of how to express his or her ideas completely in speech. Concerning the children with DS, the gestures also seems to be predictive of lexical development, but only regarding receptive vocabulary, while the specific difficulties reported concerning the transition from gesture-word combinations to multi-word utterances have been speculated as an early indicative of later deficits in syntax development, frequently reported in this population. Besides that, the studies in this field have suggested that the characteristics of the parent-child communicative interaction in children with DS, such as the production of brief and unclear gestures by the child, and the lesser responsivity of their mothers, can also contribute to the deficits in expressive language presented by these children.
\end{abstract}

Keywords: Gestures; Development; Oral language; Children; Down syndrome

\title{
REFERÊNCIAS
}

1. Iverson J, Thal D. Communicative transitions: there's more to the hands than meet the eyes. In: Wetherby AM, Warren SF, Reichle J, editors. Transitions in prelinguistic communication. Baltimore: Paul H. Brookes; 1998.

2. Bates E, Thal D, Whitesell K, Fenson L, Oakes L. Integrating language and gesture in infancy. Dev Psychol. 1989;25(6):1004-19.

3. Capone NC, McGregor KK. Gesture development: a review for clinical and research practices. J Speech Lang Hear Res. 2004;47(1):173-86.

4. McEachern D, Haynes WO. Gesture-speech combinations as a transition to multiword utterances. Am J Speech Lang Pathol. 2004;13(3):227-35.

5. Ozçaliskan S, Goldin-Meadow S. Do parents lead their children by the hand? J Child Lang. 2005;32(3):481-505.

6. Ozçaliskan S, Goldin-Meadow S. When gesture-speech combinations do and do not index linguistic change. Lang Cogn Process. 2009;24(2):190.

7. Rowe ML, Goldin-Meadow S. Early gesture selectively predicts later language learning. Dev Sci. 2009;12(1):182-7.

8. Iverson JM, Longobardi E, Caselli MC. Relationship between gestures and words in children with Down's syndrome and typically developing children in the early stages of communicative development. Int J Lang Commun Disord. 2003;38(2):179-97.

9. Iverson JM, Goldin-Meadow S. Gestures paves the way for language development. Psychol Sci. 2005;16(5):367-71.

10. Rowe ML, Goldin-Meadow S. Differences in early gesture explain SES disparities in child vocabulary size at school entry. Science. 2009;323(5916):951-3.
11. Rowe ML, Ozçaliskan S, Goldin-Meadow S. Learning words by hand: Gesture's role in predicting vocabulary development. First Lang. 2008;28(2):182-99.

12. Brooks R, Meltzoff AN. Infant gaze following and pointing predict accelerated vocabulary growth through two years of age: a longitudinal, growth curve modeling study. J Child Lang. 2008;35(1):207-20.

13. Thal DJ, Tobias S. Communicative gestures in children with delayed onset of oral expressive vocabulary. J Speech Hear Res. 1992;35(6):1281-9.

14. Hill EL, Bishop DV, Nimmo-Smith I. Representational gestures in developmental coordination disorder and specific language impairment: Error-types and the reliability of ratings. Hum Mov Sci.1998;17(45):655-78

15. Sawer E, Levine SC, Goldin-Meadow S. Early gesture predicts language delay in children with pre- or perinatal brain lesions. Child Dev. 2010;81(2):528-39.

16. Cook SW, Goldin-Meadow S. The role of gesture in learning: do children use their hands to change their minds? J Cogn Dev. 2006;7(2):211-32.

17. Chan JB, Iacono T. Gesture and word production in children with Down syndrome. Augment Altern Commun. 2001;17(2):73-87.

18. Porto E, Limongi SCO, Santos IG, Fernandes FDM. Amostra de filmagem e análise da pragmática na síndrome de Down. Pró-Fono. 2007;19(2):159-66. 
19. Andrade RV, Limongi SCO. A emergência da comunicação expressiva na criança com síndrome de Down. Pró-Fono. 2007;19(4):387-92.

20. Stoel-Gammon C. Down syndrome phonology: Developmental patterns and intervention strategies. Downs Syndr Res Pract. 2001;7(3):93-100.

21. Fabbretti D, Pizzuto E, Vicari S, Volterra V. A story description task in children with Down's syndrome: lexical and morphosyntactic abilities. J Intellect Disabil Res. 1997;41(Pt 2):165-79.

22. Franco F, Wishart JG. Use of pointing and other gestures by young children with Down syndrome. Am J Ment Retard. 1995;100(2):160-82.

23. Singer Harris NG, Bellugi U, Bates E, Jones W, Rosen M. Contrasting profiles of language development in children with Williams and Down Syndromes. Dev Neuropsychol. 1997;13(3):345-70.

24. Caselli MC, Vicari S, Longobardi E, Lami L, Pizzoli C, Stella G. Gestures and words in early development of children with Down syndrome. J Speech Lang Hear Res. 1998;41(5):1125-35.

25. Stefanini S, Caselli MC, Volterra V. Spoken and gestural production in a naming task by young children with Down syndrome. Brain Lang. 2007;101(3):208-21.

26. Zampini L, D'Odorico L. Communicative gestures and vocabulary development in 36-month-old children with Down's syndrome. Int J Lang Commun Disord. 2009;44(6):1063-73.

27. Wagner SM, Nusbaum H, Goldin-Meadow S. Probing the mental representation of gesture: Is handwaving spatial? J Mem Lang. 2004;50(4):395-407.

28. Ozçaliskan S, Goldin-Meadow S. Gesture is at the cutting edge of early language development. Cognition. 2005;96(3):B101-13.

29. Cleland J, Wood S, Hardcastle W, Wishart J, Timmins C. Relationship between speech, oromotor, language and cognitive abilities in children with Down's syndrome. Int J Lang Commun Dis. 2010;45(1):83-95.

30. Iverson JM, Capirci O, Volterra V, Goldin-Meadow S. Learning to talk in a gesture-rich world: Early communication in Italian vs. American children. First Lang. 2008;28(2):164-81.
31. Berglund E, Eriksson M, Johansson I. Parental reports of spoken language skills in children with Down syndrome. J Speech Lang Hear Res. 2001;44(1):179-91.

32. Ypsilanti A, Grouios G, Alevriadou A, Tsapkini K. Expressive and receptive vocabulary in children with Williams and Down syndromes. J Intellect Disabil Res. 2005;49(Pt 5):353-64.

33. Flabiano FC, Limongi SCO. Relação entre os gestos e a linguagem oral em um par de gêmeos com Síndrome de Down. Rev Soc Bras Fonoaudiol. 2006;11(2):116-23.

34. Goldin-Meadow S, Goodrich W, Sauer E, Iverson J. Young children use their hands to tell their mothers what to say. Dev Sci. 2007;10(6):77885.

35. Voivodic MAMA, Storer MRS. O desenvolvimento cognitivo das crianças com síndrome de Down à luz das relações familiares. Pscol Teor Prát. 2002;4(2):31-40.

36. Warren SF, Yoder PJ. Facilitating the transition from preintentional to intentional communication. In: Wetherby AM, Warren SF, Reichle J, editors. Transitions in prelinguistic communication. Baltimore: Paul H. Brookes; 1998

37. Slonims V, McConachie H. Analysis of mother-infant interaction in infants with down syndrome and typically developing infants. Am J Ment Retard. 2006;111(4):273-89.

38. Crais E, Douglas DD, Campbell CC. The intersection of the development of gestures and intentionality. J Speech Lang Hear Res. 2004;47(3):678-94.

39. Crais ER, Watson LR, Baranek GT. Use of gesture development in profiling children's prelinguistic communication skills. Am J Speech Lang Pathol. 2009;18(1):95-108.

40. Roberts JE, Price J, Malkin C. Language and communication development in Down syndrome. Ment Retard Dev Disabil Res Rev. 2007;13(1):26-35. Review. 